[pp.121-140]

Vol. 4(1), Enero - Junio, 2016

http://dx.doi.org/10.15359/rnh.4-1.9

\title{
El voluntariado de la Cátedra del Adulto Mayor de La Universidad de La Habana: sus motivos esenciales
}

Older People Chair's Volunteering at the University of Havana: Its Essential Motivations

Carlos Luis Semino Garcia

Universidad de la Habana

Cuba

carlosluis.semino@psico.uh.cu

\section{Resumen}

El presente trabajo pretende caracterizar los motivos que orientan a un grupo de adultos mayores a participar como voluntarios en la Cátedra Universitaria del Adulto Mayor de La Habana (CUAM). Se identifican los motivos generales y los motivos asociados a la participación como voluntarios; se describe su jerarquía y se explora el grado de satisfacción con su desempeño a partir de sus motivaciones esenciales asociadas al proyecto. La ausencia de estudios sobre el voluntariado y la importancia de este proyecto educativo para los adultos mayores constituyen las motivaciones fundamentales para este artículo. Este es un estudio cualitativo que responde a un diseño transversal exploratorio y emplea el método clínico al recurrir a técnicas de expresión abierta e indirecta: técnica "Cinco deseos" y "Cinco deseos vinculados con la labor en el voluntariado de la CUAM"; "Técnica de satisfacción con la actividad como voluntario"; "Composición 'Trabajo en la cátedra porque..."; y la "Entrevista individual semiestructurada sobre el voluntariado". El grupo de estudio estuvo compuesto por 21 adultos mayores, miembros del consejo técnico ampliado de la CUAM, que participan como voluntarios en el rol de coordinador. Los motivos generales giran en torno a la familia, salud, deber humanitarismo, condiciones materiales y voluntariado.

Los motivos que se asocian a la actividad del voluntariado son: crecimiento del 
proyecto de la CUAM, preparación profesional y personal, permanencia y sostenibilidad del proyecto de la CUAM, reconocimiento social, relación con otras instituciones, condiciones materiales y calidad de las actividades como voluntarios.

Palabras clave: voluntariado, Cátedra Universitaria del Adulto Mayor, motivos, adulto mayor.

\begin{abstract}
The present paper aims to characterize motivations leading a group of older adults to volunteer at the Older People Chair of the University of Havana (CUAM in Spanish). General motivations and those related to volunteering were identified. The volunteers' hierarchy is described, and the level of satisfaction with their performance is explored based on their essential motivations associated with the project. The main reasons for this article were the absence of studies on volunteering and the importance of this education project for older people. This is a qualitative research responding to a cross-cutting exploratory design, and implementing the clinical method, since it has recourse to open and indirect expression techniques: "Five wishes" technique and "Five wishes linked to volunteering at the Older People Chair of the University of Havana"; "Satisfaction with volunteering" technique; Writing about "I work for the Chair because...", and "Individual semi-structured interview about volunteering". The study group consisted of 21 older people, members of the technical council of the Older People Chair who volunteered as coordinators. General motivations related to the family, health, duty, humanitarianism, material conditions and volunteering. The motivations associated with volunteering activities were: Older People Chair Project growth, personal and professional preparation, permanence and sustainability of the project, social acknowledgment, relationship with other institutions, material conditions, and quality of activities as volunteers.
\end{abstract}

Keywords: Volunteering, Older People Chair of the University of Havana, motivations, older adult.

De lo que no cabe duda es que con la edad no se cumplirá la sentencia según la cual "a la tercera va la vencida". La tercera es y será aún más, una edad para seguir aportando a la vida social, para enriquecer el alma, para coadyuvar al logro de una mejor vida para todos. (Calviño 2013, p. 86) 


\section{Introducción}

El sector de la población cubana con 60 años o más experimenta un crecimiento relativo constante, dada la baja tasa de natalidad y la creciente esperanza de vida. Es, además, expresión del desarrollo científico-técnico, el aumento del nivel de vida, la disminución de la natalidad, el progreso en los tratamientos médicos y los avances en políticas y programas de prevención para ampliar la protección social.

Este fenómeno poblacional está alterando la estructura demográfica, y tiene y tendrá una amplia repercusión social, económica y cultural. Por tanto, es necesario afrontar esta nueva situación y, para ello, la promoción del envejecimiento activo resulta un enfoque indispensable.

El programa educativo de la Cátedra del Adulto Mayor (CUAM), de la Universidad de La Habana, constituye una red de apoyo para la implementación de las nuevas políticas que se interesan por la atención de las personas mayores. Este espacio propicia la incorporación social. Brinda apoyo emocional a través del intercambio permanente de conocimientos, de sentimientos y de expresiones afectivas. Prepara a estas personas para enfrentarse a situaciones nuevas, difíciles. Tiene como objetivo mejorar su calidad de vida, favorecer la educación continua y permanente, así como lograr un mayor bienestar psicológico.

En este contexto, los esfuerzos dirigidos a promover el voluntariado en las personas adultas mayores juegan un papel crucial. Sus propuestas e iniciativas abren puertas de participación en un contexto que se presenta como un lugar para la superación, una puerta a la interacción interpersonal, espacio para hacer y sentirse protagonistas de acciones que les otorgan satisfacciones al colaborar y brindar atención a otras personas. Posibilita que se reafirme su identidad colectiva, $\mathrm{y}$ fortalece los lazos familiares, intergeneracionales y comunitarios.

En ese sentido, este artículo se orienta a caracterizar los motivos que orientan a un grupo de personas adultas mayores a participar como voluntario de la Cátedra del Adulto Mayor de la Universidad de La Habana.

\section{Los motivos}

El estudio de los motivos atraviesa por diferentes momentos, los cuales se pueden dividir en estudios desde la psicología de orientación no marxista hasta la de orientación marxista. 
En los primeros destacan el factorialismo, el conductismo, el neoconductismo, el psicoanálisis freudiano, el humanismo y el cognitivismo. Estas concepciones presentaban algunas limitaciones para caracterizar el proceso de autorregulación del comportamiento en el sujeto (Domínguez, 2005); la explicación del comportamiento a través del modelo estímulo-respuesta que imposibilitan la comprensión de la esencia subjetiva del ser humano, con lo cual la motivación humana queda fuera del análisis psicológico; la estrecha vinculación de los motivos con el impulso biológico, que después se extiende como centro de la vida psíquica de los seres humanos, aunque estos sean o no conscientes, para el sujeto (Calviño, 1983); el énfasis en el carácter superior de la motivación humana y el papel del yo y de la conciencia en la regulación del comportamiento (Domínguez, 2005); la absolutización del papel que desempeñan los factores intelectuales en la regulación y autorregulación del comportamiento, y obvian el papel que juegan, en el potencial regulador de la motivación, los procesos afectivos de la personalidad (Martínez, 2009).

Dentro de la psicología de orientación marxista se desatacan estudios soviéticos como los de Bozhovich y sus colaboradores. Para estos, la motivación humana tiene un carácter esencialmente consciente, el cual no niega la existencia de motivos inconscientes. Esto está presente, además, en los trabajos de psicología de Rubinstein, Petrovski, Ansiférova, D. González, F, González, Calviño y demás (Domínguez, 2005).

González (1990) considera que los motivos se estructuran en una jerarquía motivacional; los que conforman el nivel superior de dicha jerarquía son aquellos que orientan la personalidad hacia sus objetivos esenciales. Estos motivos constituyen las tendencias orientadoras de la personalidad. La elaboración consciente del individuo les imprime fuerza dinámica a los motivos, por lo que se convierten en tendencias orientadoras de la personalidad cuando su potencial movilizador trasciende la situación presente del sujeto y se proyecta hacia su futuro.

En cuanto a la jerarquía de motivos, se expresa en distintos niveles y grados de complejidad. Se integran con un nivel dado de organización en la personalidad del sujeto y distingue su potencial regulador sobre el comportamiento y las formas de su expresión en dependencia de la posición que ocupan en la jerarquía de motivos de la personalidad (González, 1990). 
"La forma en que el motivo se manifiesta depende de la personalidad del sujeto que lo asume" (González, 1990, p. 116). Por ello, un mismo motivo puede acarrear fines o estrategias diferentes para su satisfacción, en un nivel más o menos racional de elaboración.

\section{El voluntariado}

La Ley Reguladora del Voluntariado Social en el Ámbito Estatal en España (Ley 6/1996, Artículo 3), entiende por voluntariado:

El conjunto de actividades de interés general, desarrolladas por personas físicas, siempre que las mismas no se realicen en virtud de una relación laboral, funcionarial, mercantil o cualquier otra retribuida y reúna los siguientes requisitos: que tengan carácter altruista y solidario; que su realización sea libre, sin que tengan su causa en una obligación personal o deber jurídico; que se lleven a cabo sin contraprestación económica, sin perjuicio del derecho al reembolso de los gastos que el desempeño de la actividad voluntaria ocasione; que se desarrollen a través de organizaciones privadas o públicas y con arreglo a programas y proyectos concretos. (Montero y Bedmar, 2010, p. 76)

Esta definición ha sido incorporada a diversos estudios sobre esta temática (Agost, et al., 2002; Ayarza, 2008; Corrall, 1996; Salvador, 2010; Yubero, S. y Larrañaga, 2002; ). De estas referencias se pueden resumir las siguientes características que presenta el voluntariado: se produce en elárea de la gestación de valores colectivos de la sociedad civil y en menor medida en el sector estatal; constituye una ayuda o servicio libre y espontáneo a las otras personas sin que sea, ni exclusiva ni primordialmente, en beneficio de quien la desarrolla, la persona debe poseer un compromiso estable a permanecer en la actividad para considerarse voluntaria; estas acciones deben gestarse en el marco de una organización con independencia del grado de institucionalización, reconocimiento público o privado que esta tenga; no está mediada por una relación laboral, funcional, ni mercantil y se produce gracias a un gran activismo y compromiso con el contexto social, como acción que repercute en el bienestar de la comunidad. Estos estudios concuerdan en definir el voluntariado como una pieza fundamental para la construcción de la sociedad civil. 


\section{El envejecimiento poblacional en Cuba}

La tendencia hacia el envejecimiento de la población acarrea profundos cambios en todas las generaciones y en la mayoría de las actividades sociales, económicas y políticas. Durante el siglo XXI Cuba ha estado marcada por una sociedad más envejecida, tópico que se ha convertido en asunto de gran relevancia para la protección social, el mercado de trabajo, la política, el diseño y la tecnología, la educación y la cultura.

Este cambio implica retos importantes para instituciones y políticas educativas que han surgido para la atención a este sector de la población, entre las que destaca la Cátedra Universitaria del Adulto Mayor.

Este proyecto se apoya, para su operacionalización, en un grupo de personas que desempeñan diversos roles de forma voluntaria. Por sus peculiaridades, este movimiento se conoce con el nombre de voluntariado, el cual es conocido a nivel mundial. Existen modelos para su estudio desde diferentes perspectivas de la ciencia encaminados a comprender aspectos tales como: las motivaciones de los sujetos voluntarios, la permanencia dentro del voluntariado, el sentido de la participación en la tarea, entre otros.

Proliferan discusiones respecto a alternativas metodológicas a seguir para la caracterización de este fenómeno, y existen variantes que abogan por el uso de metodologías cuantitativas (uso de cuestionarios y escalas cerradas); hasta las más recientes que se pronuncian por el uso de metodologías cualitativas, con su traducción en métodos e instrumentos de carácter abierto.

A pesar de la probada importancia del voluntariado como "recurso" social (García Roca y Comes Ballester, 1995), en Cuba, no existen investigaciones previas en la Cátedra Universitaria del Adulto Mayor, que ya celebra sus 16 años de existencia. Por lo tanto, esta investigación constituye un primer acercamiento a esta temática que no ha recibido la atención científica que requiere.

Se retoman como antecedentes los estudios sobre esta temática existentes en países como España, Uruguay y Argentina que consideran las elaboraciones teóricas y metodológicas previas, para defender como punto de partida, el uso de una metodología cualitativa, con el empleo del método clínico y de técnicas abiertas, que pretende un ajuste de los indicadores a la realidad del fenómeno de acuerdo con cómo se ha producido el surgimiento y evolución del proyecto de la CUAM. 
Este voluntariado constituye una de las experiencias de este tipo que existen en Cuba. Es, a su vez, uno de los movimientos sociales de mayor alcance y trascendencia por la importancia que reviste para el funcionamiento de las aulas de la CUAM en cada municipio de La Habana. El voluntariado es una modalidad que opera con nuevos retos, por lo que resulta de interés significativo conocer su peso social y sus motivaciones -con las promesas y riesgos que acarrea- para gestionarlo y obtener de él la potencialidad de regeneración que posee.

Los resultados de esta investigación podrán utilizarse para el rediseño de las políticas y estrategias a seguir para la atención al voluntariado desde los distintos niveles de decisión y organización de las actividades en las cuales laboran, por lo que posee una utilidad práctica.

\section{Método}

\section{Población y grupo de estudio}

La población está compuesta por las personas adultas mayores de los municipios de La Habana, que participan como voluntarias en diversos roles: coordinadoras de municipios, presidentas de aula y profesoras y colaboradoras de la docencia; para un total de 309. El grupo quedó conformado de forma intencional, con 21 sujetos adultos mayores, miembros del Consejo Técnico Ampliado de la CUAM, que participan como voluntarios en el rol de Coordinador General en varios municipios de La Habana. Se tuvo en cuenta que los sujetos tuvieran 60 años o más de edad; pertenecer y participar en la CUAM como parte del Consejo Técnico Ampliado; encontrarse trabajando en el rol de coordinador de municipio de la provincia La Habana; y la disposición a participar en el estudio. 
Tabla 1

Información sociodemográfica de la población participante

\begin{tabular}{llr}
\hline Variables sociodemográficas & Porcentaje \\
\hline \multirow{2}{*}{ Sexo } & Femenino & 95,2 \\
& Masculino & 4,8 \\
\hline \multirow{3}{*}{ Escolaridad } & Universitaria & 76,2 \\
& Técnico medio & 14,3 \\
& 12mo grado & 9,5 \\
\hline \multirow{4}{*}{ Estado civil } & Casada & 14,3 \\
& Viuda & 38,1 \\
& Divorciada & 33,3 \\
& Soltera & 9,5 \\
\multirow{2}{*}{ Ocupación } & Unión consensual & 4,8 \\
& Jubilada & 85,0 \\
& Otras ocupaciones & 13,0 \\
\hline
\end{tabular}

Nota: Elaboración propia.

\section{Objetivos}

Para el acercamiento a este grupo, la investigación persigue, como objetivo general, caracterizar los motivos que orientan a un grupo de personas adultas mayores a participar como voluntarias de la Cátedra del Adulto Mayor de la Universidad de La Habana.

Específicamente, se busca identificar los motivos generales de esta población adulta mayor y los motivos asociados a su participación en el voluntariado en la CUAM; describir la jerarquía de los motivos generales y de los motivos asociados a su participación voluntaria en la CUAM; explorar su grado de satisfacción con el desempeño como personal voluntario de la CUAM a partir de sus motivaciones esenciales asociadas a este proyecto.

\section{Tipo de investigación}

Se emplea una metodología esencialmente cualitativa. Responde a un diseño transversal de carácter exploratorio descriptivo. Recurre principalmente a técnicas de expresión abierta e indirectas para conocer las características esenciales 
que presenta la esfera motivacional en el grupo de personas adultas mayores voluntarios estudiados.

Esta metodología contribuye a la aproximación al objeto de estudio para revelar sus cualidades esenciales, describir e interpretar los fenómenos y conocer, en este caso, los procesos complejos que constituyen la subjetividad de la persona adulta mayor voluntaria, y se evade el instrumentalismo y las rutinas clasificatorias.

Se privilegia el método clínico, abordado mediante una serie de técnicas que estudian la individualidad de forma sistemática e intensiva. Al mismo tiempo se hace posible la construcción de un sistema de categorías para el análisis de los resultados a través del método de análisis de contenido.

Los análisis eminentemente cualitativos se apoyan en procedimientos estadísticos como el cálculo porcentual y se utilizan estadísticos descriptivos de frecuencia (la moda y la media), como medidas de tendencia central.

"La epistemología cualitativa defiende el carácter constructivo-interpretativo del conocimiento, lo que de hecho implica comprender el conocimiento como una producción y no como la aprehensión lineal de una realidad que se nos devela" (González, F. 2009, p. 4). Para ello, los instrumentos son implementados en pos de la construcción del conocimiento, cuidando de incurrir en procesos clasificatorios.

\section{Instrumentos}

Técnicas "Cinco deseos" y "Cinco deseos vinculados con la labor en el voluntariado de la CUAM". Se reconoce el origen de esta técnica en el belga Joseph Nuttin. Fue retomada en la propuesta que sobre ella hizo, en el año 1972, el doctor Diego González Serra. En la presente investigación se aplica una variante de esta técnica donde, en un primer momento, se enfoca en la exploración de los motivos de forma general ("Cinco deseos") y en un segundo momento, se aplica para explorar los motivos que orientan la actividad específica ("Cinco deseos vinculados con la labor en el voluntariado de la CUAM").

Para el análisis de contenido de los "Cinco deseos" se utilizarán, como categorías de referencia, las empleadas por González (2008) y ajustes posteriores que aparecen en varias tesis de diploma (Águila, 2004; Estévez, 2008; Martínez, 2007; Paucar, 2009; Suárez, 2007). A partir de las anteriores propuestas y de acuerdo con los datos obtenidos, se construyeron las categorías que aparecen a continuación, siguiendo los principios que rigen un estudio esencialmente cualitativo. Estas categorías son las siguientes: 
Familia

Relaciones de pareja

Salud

Realización de sí mismo o misma

Relaciones interpersonales

Deber- humanitarismo

País
Condiciones materiales

Recreación

Viajar

Voluntariado

Trascendencia

Necesidades afectivas

Para analizar el contenido expresado en la técnica de los "Cinco deseos vinculados con la labor en el voluntariado de la CUAM", se consideran las mismas referencias que para la técnica de los "Cinco deseos" y se procedió con la misma perspectiva metodológica que en el caso anterior. Las categorías construidas fueron las siguientes:

$\begin{array}{ll}\text { Preparación profesional y personal } & \text { Calidad de sus actividades de voluntario } \\ \text { Relaciones Interpersonales } & \text { Relación con instituciones } \\ \text { Condiciones materiales } & \text { Crecimiento del proyecto de la CUAM } \\ \text { Deber-humanitarismo } & \text { Permanencia del proyecto de la CUAM } \\ \text { Trascendencia } & \text { Salud } \\ \text { Reconocimiento social del programa } & \text { Viajar }\end{array}$

Técnica de satisfacción con la actividad como voluntario. Se emplea esta técnica para conocer el grado de satisfacción de las personas adultas mayores con su desempeño dentro de la CUAM como voluntarias. Aunque su empleo está más vinculado al ámbito organizacional, resulta eficaz para explorar las perspectivas del grupo de estudio con su labor. Se aplica de modo independiente y en el análisis la medida que se utilizará es la moda, medida de tendencia central que indica el valor más frecuente o repetido en la matriz de respuestas ofrecidas. Para ello se refiere una escala (Cuestay Valencia, 2014) desde el 1 hasta el 3, donde 1 significará la mejor percepción de la satisfacción y 3 significará la peor percepción de la satisfacción. Se emplean los siguientes indicadores:
Reconocimiento moral
Sobrecarga de tareas
Utilidad y aporte social
Experiencia en el desempeño como voluntarios o voluntarias
Bienestar emocional 
Preparación para la

adultez mayor

Desarrollo de cualidades

personales

Acceso a actividades

recreativas

Composición: "Trabajo en la cátedra porque..." El análisis integral de esta técnica permite el estudio de los diferentes motivos, intereses y necesidades de la personalidad. Para este fin, se realiza el análisis de los contenidos manifestados por los sujetos, teniendo en consideración la gama de intereses y motivaciones, el grado de implicación afectiva, es decir, si estaban simplemente expuestos, descritos, o si se expresa un compromiso afectivo, vinculado emocionalmente con el material. El uso e interpretación de esa técnica parte de los principios metodológicos expuestos por González Rey (1985). Para el análisis de la composición se consideraron tres aspectos fundamentales: contenido, vínculo emocional hacia el contenido y elaboración personal del contenido expresado.

Entrevista individual semiestructurada sobre el voluntariado. Se realizó una entrevista semiestructurada con el objetivo de tener una visión más integradora de la población mayores estudiada. Se indaga sobre los contenidos relacionados con los objetivos específicos de esta investigación; en lo que respecta a sus principales contenidos psicológicos: motivos, necesidades, actitudes, tareas o acciones que realizan en el voluntariado, satisfacción con dicha labor; así como para integrar indicadores obtenidos en las técnicas de expresión escrita anteriormente aplicadas. Como parte de la entrevista, se realiza una observación de la dinámica generada durante su aplicación. Se valora la expresión corporal y emocional de los sujetos, el grado de adecuación a lo expresado verbalmente. Para su evaluación se tuvieron en cuenta los indicadores definidos para explorar los motivos asociados a la actividad de voluntariado, el conocimiento acerca de la actividad y el grado de satisfacción experimentado.

\section{Procedimiento}

En la primera sesión se estableció el "Rapport", el cual había sido promovido desde la propia relación del sujeto investigador con los sujetos voluntarios en los espacios de coordinación que se desarrollan en la Cátedra. Se plantearon los objetivos del estudio, su pertinencia y alcance de los resultados previstos y se 
contó con el consentimiento de los sujetos para participar en la investigación. En esta sesión se procedió a la aplicación de la técnica de los cinco deseos, la técnica de satisfacción y la composición. Posteriormente se procedió a desarrollar la interpretación de la información recogida en estas técnicas de "papel y lápiz" y a la elaboración de resultados parciales.

Sobre la base de los avances derivados en la interpretación de los resultados, se procedió a la realización de la entrevista individual. Para ello se hicieron las coordinaciones pertinentes para el encuentro individual de los sujetos. Se aplicó finalmente la entrevista, que permitió profundizar, desde el punto de vista dialógico e interactivo, en aquellos aspectos que no quedaron totalmente develados durante la aplicación de las técnicas anteriores.

\section{Discusión de los resultados}

Al analizar de forma integrada y global los resultados obtenidos en la investigación, vemos que los sujetos adultos mayores investigados se caracterizan por poseer un buen número de motivos que conforman su jerarquía motivacional. Este hecho resulta significativo, pues la presencia de motivos a nivel de la personalidad ofrece direccionalidad al comportamiento de estos sujetos y los impulsa a la obtención de metas de desarrollo en un sentido amplio, lo que confirma la perspectiva de que el desarrollo humano es un proceso continuo que se extiende a lo largo de todo el ciclo vital.

Desde el propio planteamiento inicial de los motivos generales emergen motivos relacionados con la actividad que desempeñan como voluntarios y voluntarias. Tal es el caso del motivo deber-humanitarismo, que es reconocido ampliamente dentro de la bibliografía científica sobre el voluntariado, como un motivo que orienta la conducta de las personas que se desempeñan en este tipo de actividades.

El voluntariado como elemento de la estructura social es un movimiento que, al tener la característica de no ser remunerado, los sujetos que lo integran trabajan a partir de una motivación genuina y auténtica. Su hacer se orienta por un alto sentido del deber, la creación de nuevos valores humanos y la aplicación de valores universales como la solidaridad humana, la paz, el altruismo y la preservación del bien común para todos los grupos.

Dada la significación que tiene la condición de voluntariado en sus propias vidas, esa condición misma se revela, desde el inicio de la investigación, como uno de 
los motivos esenciales de estas personas adultas mayores. Se puede inferir que este motivo funciona como uno de los principales que orienta la conducta de estas personas en el presente, y que influye en la dinámica de la personalidad en las distintas esferas de la vida, si se parte de considerar que los contenidos que integran la personalidad funcionan de un modo sistémico.

En cuanto a los motivos generales que aparecen, en correspondencia con lo planteado por la bibliografía, la primacía de motivos relacionados con la familia y la salud están en correspondencia con las significaciones que adquieren estos aspectos de la situación social de su desarrollo, pues la familia constituye uno de los escenarios fundamentales de apoyo y contención para el sujeto al arribar a esta etapa de la vida. Por otro lado, las ideas sobre la salud y la perspectiva de la vida se derivan de duelos que debe elaborar el sujeto adulto mayor, temas que ocupan un lugar frecuente dentro de sus reflexiones cotidianas.

Resulta interesante el hecho de que, aunque se hacen referencias a estos motivos en sentido general, al argumentarlos se utilizan elementos donde la condición de voluntariado tiene cierto grado de centralidad. Es decir, los propios contenidos de los motivos que aparecen reflejados en sentido general, están matizados por la condición de ser voluntarios o voluntarias.

Con respecto a los motivos asociados a la actividad que desarrollan las personas adultas mayores como voluntarias, igualmente se presentan un número importante de ellos, casi comparable a la cantidad de motivos planteados de modo general por los sujetos. Esto indica una gran variedad de contenidos que movilizan el comportamiento de los sujetos, para participar en las actividades que realizan como voluntarios dentro del proyecto de la cátedra.

Se observa una alta implicación personal con estos contenidos que denotan una fuerte identificación con el proyecto de la CUAM, que se manifiesta directamente en el deseo prioritario de que progrese, mantenga su permanencia, se sostenga en el tiempo como proyecto y que adquiera cada vez más reconocimiento social. Para el logro de este, asociado a una mayor legitimidad a nivel comunitario y social, las personas adultas mayores visualizan de forma certera la importancia que tendría contar con un mayor apoyo gubernamental y de los distintos actores sociales con protagonismo en nuestra sociedad civil.

Esto derivaría en una mejor relación con otras instituciones del Estado, organizaciones de masas y espacios comunitarios. Maximizaría los alcances de 
la CUAM y su impacto como proyecto que promueve el desarrollo humano en una de las etapas más sensibles del ciclo vital, como lo es la vejez.

Esta participación dentro de la CUAM, de voluntariado, no se ve influenciada por la obtención de remuneración económica. Tal y como se manifiesta en los estudios sobre el voluntariado descritos en revisión teórica de este informe, en este caso, reciben la gratificación de que el proyecto influye en su preparación profesional y personal. En cuanto a lo profesional se destaca el desarrollo de competencias. Además, se reconoce la ganancia subjetiva personal de trabajar en un proyecto que tributa al desarrollo de cualidades personales desarrolladoras.

En el grupo de estudio existe un fuerte compromiso con la calidad de las actividades que desarrollan. Posee una mirada crítica que va a la búsqueda de niveles superiores de perfeccionamiento en aspectos intrínsecos a la actividad de voluntariado, lo cual tributa al reconocimiento de este programa en cuanto a los resultados que se expresan en el sector poblacional al cual va dirigido.

Como aspecto peculiar se pudo comprender, sobre todo en las técnicas de expresión abierta y en las de carácter dialógico, como la entrevista, la significación que posee la categoría deber-humanitarismo, la cual está presente en los motivos de los sujetos. Esta se asocia con la finalidad de la labor que realizan en pos del desarrollo humano y la defensa de valores importantes.

Aparece también en estas técnicas la categoría trascendencia en correspondencia con una de las regularidades de estas edades, pues se corresponde con la nueva formación psicológica, centro de la dinámica del desarrollo en la persona adulta mayor, lo cual implica que los procesos del desarrollo en la etapa están estrechamente vinculados con el desarrollo de esta formación.

En la exploración de los motivos asociados al voluntariado aparecen otras categorías que se catalogaron como "otras", entre las cuales emergen elementos identitarios en los sujetos. A partir de ello se infiere que se ha comenzado a conformar una identidad como voluntarios o voluntarias en el grupo estudiado.

Como esta investigación se desarrolla desde una perspectiva esencialmente cualitativa, el diseño se ajustó para profundizar en aquellas características que pudieran estar configurando una identidad como voluntarios y voluntarias en los sujetos del grupo de estudio. Se obtuvo así un grupo de elementos expresados por 
el grupo que permitieron establecer vínculos con las actividades que realizan y los niveles de satisfacción que se logran desde sus motivaciones esenciales.

Entre las características más importantes referidas que podrían configurar una identidad en el grupo como voluntario, se encuentran, desde las expresiones de los sujetos, que consideran voluntaria a la persona que desarrolla esta actividad sin retribución económica. Esta debe poseer valores humanos como el altruismo, la solidaridad, la responsabilidad y el sentido del deber, entre otros. Consideran que son sujetos promotores del desarrollo humano, y destacan en sus argumentos la importancia que le conceden a la influencia que pueden ejercer en aspectos como su autoestima y su autoperfeccionamiento. Refieren que quien ejerce el voluntariado es una persona que necesita mantener la vitalidad y los intereses personales hacia distintas esferas del saber, necesita un tiempo mínimo de permanencia en las actividades y es una persona que aprende y se autoperfecciona. Por último, aparecen cualidades morales elevadas, pues se vivencia con vinculación a la defensa de la Revolución.

Como acciones más significativas de los sujetos voluntarios se encuentran las desarrolladas para la socialización de los resultados del programa, la gestión del personal para impartir los módulos y aquellas encaminadas a garantizar las condiciones materiales mínimas indispensables para las actividades.

Si se tienen en cuenta sus contenidos, estas actividades pudieran estar relacionadas con la formación de los elementos identitarios referidos con anterioridad. Como formadoras de sentidos personales, pudieran estar vinculadas con la existencia de motivos más relevantes de los sujetos en esta área, es decir, se estructuran a nivel subjetivo los motivos más relevantes asociados con el rol de voluntarios o voluntarias. A su vez, en la medida en que existen motivos que poseen relevancia para estos sujetos, ellos conducen al desarrollo de actividades de mayor calidad dentro de la cátedra.

De esta forma, el motivo asociado al crecimiento del proyecto, su permanencia y sostenibilidad, así como su reconocimiento social, se ve favorecido por las actividades que implican la socialización de sus resultados. Estas, a su vez, refuerzan la motivación en esta línea.

Además, la selección de personal con la capacidad adecuada impacta la motivación hacia el logro de una preparación profesional y personal. Los nuevos niveles 
de motivación que se obtienen en esta área, se presume, llevarán a un mejor desarrollo de estas actividades, al buscar nuevos niveles de perfeccionamiento.

El análisis de los niveles de satisfacción en relación con las motivaciones esenciales en su participación en el voluntariado dentro del proyecto de la CUAM corrobora que la mayoría se encuentra satisfecha.

Entre las razones más importantes de dicha satisfacción se encuentra que la utilidad y aporte social constituye el indicador más importante de satisfacción en las personas adultas mayores con la actividad que realizan como voluntarias. Se relaciona con la mayoría de los motivos que orientan la actividad de estos sujetos, entre los cuales está el crecimiento del proyecto de la CUAM. Posee estrecha relación con el deseo referido a la permanencia del proyecto de la CUAM, con el reconocimiento social del programa derivado de sus éxitos hacia la comunidad y con la calidad de las actividades que desarrollan como sujetos voluntarios, lo que conduce a un mayor reconocimiento. Esta satisfacción encuentra su realización a través del desarrollo de acciones para la socialización de los resultados del programa educativo en diferentes instancias de la sociedad.

Las razones que implican satisfacción también se asocian al bienestar emocional que sienten los sujetos y derivan de la preparación profesional y personal que obtienen. También se ven favorecidas por las relaciones interpersonales y el contacto intergeneracional generador de vivencias positivas, y por el hecho de permanecer dentro del proyecto, el cual consideran que tiene una larga vida. Esta satisfacción se obtiene en estrecho vínculo con las actividades que desarrollan para la gestión del personal que imparte los módulos y la garantía de condiciones materiales adecuadas que garanticen una formación de calidad.

La satisfacción se considera influenciada por el desarrollo de cualidades personales. Se exhibe una estrecha relación entre este, como razón que implica satisfacción en los adultos mayores, y la presencia de motivos en ellos, como es el caso de las relaciones interpersonales y la preparación profesional y personal.

El reconocimiento moral es una razón asociada a la satisfacción. Trabajar por la obtención de nuevos niveles de reconocimiento social del programa les genera vivencias satisfactorias. 
Las dos razones de satisfacción mencionadas con anterioridad encuentran gratificación en las actividades vinculadas con la socialización de los resultados del trabajo que se desarrolla en el programa.

Por último, como elemento que demuestra satisfacción, se encuentra la preparación para la adultez mayor. Este es un argumento que aparece vinculado de forma estrecha con el motivo de preparación profesional y personal. Consideran que el programa les prepara en un grupo de ámbitos y genera saberes necesarios para poder enfrentar, de un modo más saludable, el proceso de envejecimiento. Se ve favorecida por la selección del personal adecuado para realizar una capacitación en estos aspectos imprescindibles para su desarrollo.

A partir de los resultados obtenidos en la investigación, se puede resumir que:

- Los cinco motivos más referidos por lo sujetos, de modo general, se relacionan con: familia, salud, deber-humanitarismo, condiciones materiales y voluntariado.

- Los motivos más relevantes en el grupo estudiado, que se asocian a la actividad del voluntariado, son: crecimiento del proyecto de la CUAM, preparación profesional y personal, permanencia y sostenibilidad del proyecto de la CUAM, reconocimiento social, relación con otras instituciones, condiciones materiales y calidad de las actividades como voluntarios y voluntarias.

- La jerarquía de motivos generales se comporta en el siguiente orden: familia, salud, deber-humanitarismo y condiciones materiales.

- La jerarquía de motivos asociados al voluntariado se comporta en el siguiente orden: crecimiento del proyecto de la CUAM, relación con instituciones, condiciones materiales, reconocimiento social y calidad de las actividades desarrolladas como sujetos voluntarios.

- El grado de satisfacción predominante en las personas adultas mayores, con el desempeño como voluntarias dentro de la CUAM, a partir de sus motivos esenciales es: satisfecha.

- Los argumentos asociados con este estado de satisfacción están relacionados con: la utilidad y el aporte social del proyecto de la CUAM, el bienestar emocional derivado de la participación dentro del proyecto, el desarrollo de cualidades personales favorecidas por la condición de ser personas 
voluntarias, el reconocimiento moral alcanzado y la preparación para la adultez mayor, que favorece el tránsito por esta etapa de forma más saludable.

- La utilidad y el aporte social se relacionan con la mayoría de los motivos asociados a la participación como personas voluntarias dentro del proyecto. En particular, se vincula con la permanencia del proyecto de la CUAM, con el reconocimiento social del programa y con la calidad de las actividades que desarrollan como sujetos voluntarios.

- El bienestar emocional que sienten los sujetos, se vincula con la preparación profesional y personal, y las relaciones interpersonales. Esto ocurre también con la categoría referida a la satisfacción cualidades personales y los motivos relaciones interpersonales y preparación profesional y personal.

- El reconocimiento moral resulta una razón de satisfacción.

- Finalmente, la preparación para la adultez mayor se encuentra vinculada con el motivo de preparación profesional y personal.

\section{Referencias}

Agost, M. R., Fernández, V., Martínez, J. y Nebot, E. (2002). ¿Por qué decidimos ser voluntarios?. Jornades de Foment de la Investigació, Universitat Jaume. Recuperado de http://repositori.uji.es/xmlui/bitstream/handle/10234/79720/ Forum_2002_36.pdf? sequence $=1$

Ayarza Elorriaga, E. (2008). II Plan Vasco del Voluntariado: ejes fundamentales. Revista de servicios sociales, S.V., (44), 127-134. Recuperado de http://www.redalyc.org

Calviño, M. (1983) Selección de Lecturas de motivación y procesos afectivos. (Tomo 2). La Habana: MES.

Calviño, M. (2013). Vale la Pena. Escritos con Psicología. La Habana: Caminos.

Carmona, L. (2013). Influencia del programa educativo de la Cátedra Universitaria del Adulto Mayor en la percepción de la calidad de vida de un grupo de cursantes combatientes (Tesis de Diploma). La Habana, Cuba.

Corrall, F. B. (1996). Atado, mudo y bastante arrugado ¿Existen nuevas formas y conceptos de voluntariado? Revista Documentación Social, S.V., (104), 103-114. 
Domínguez García, L. (2005). Motivación profesional y personalidad. En: Lourdes Fernández Ruíz (Comp.), Pensando en la personalidad. La Habana: Félix Varela.

García Roca, J. y Comes Bellester, J. A. (1995). El voluntariado como recurso social. Premio Bancaixa de investigación sobre Servicios Sociales 1994. Valencia: Bancaixa.

González Rey, F. L. (1985). Psicología de la personalidad. La Habana, Cuba: Pueblo y Educación.

González Rey, F. L. (1990). Psicología de la Personalidad. La Habana, Cuba: Pueblo y Educación.

González Rey, F. L. (2009). Investigación cualitativa y subjetividad. Los procesos de construcción de la información. México D. F.: McGraw-Hill.

González Serra, D. J. (2008). Psicología de la motivación. La Habana, Cuba: Ciencias Médicas.

Ladrón de Guevara, B. (1994). Antropología de la vejez. Literal. IV (20) 45-47.

López Navas, A. y Ortigosa, J. M. (2014). Teorías sobre el envejecimiento. En Teresa Orosa Fraíz (Coord.) Temas de Psicogerontología. La Habana, Cuba: UH.

Montero García, I. y Bedmar Moreno, M. (2010). Ocio, tiempo libre y voluntariado en personas mayores. Revista Polis de la Universidad Bolivariana, 9, (26), 61-84. Recuperado de http://polis.revues.org/

Salvador Hernández, P. P. (Coord.) (2010). Manual para la formación del voluntariado. Castilla - La Mancha. España: Consejería de Salud y Bienestar Social. Recuperado de http://www.redalyc.org

Schupack, S.; Álvarez, M. J.; López, A.; Molina, S. y Martínez, G. (2014). La participación de Adultos Mayores en Voluntariado Social: Una herramienta para la autonomía. V Jornadas Distritales de Psicología del Oeste, Argentina, pp. 29-30.

Valencia Rodríguez, Liliana y Velandia Morales, Andrea. (2013). Percepciones y 
motivaciones sobre el voluntariado. Revista Eleuthera, 9 (2), 116-131. Recuperado de http://eleuthera.ucaldas.edu.co/downloads/Eleuthera9_8.pdf

Yubero, S. y Larrañaga, E. (2002). Concepción del voluntariado desde la perspectiva motivacional: conducta de ayuda vs altruismo. Revista Pedagogía Social, 2 (9), 27-39. Recuperado de http://www.redalyc.or.or 